\title{
Giant primary scrotal lipoma: A case report
}

\author{
Massimiliano Creta $^{1}$, Giacomo De Stefano ${ }^{2}$, Roberto Buonopane ${ }^{1}$, Ciro Barba ${ }^{2}$, Sergio Di Meo ${ }^{1}$, \\ Vittorio Imperatore $^{1}$, Ciro Imbimbo ${ }^{3}$, Vincenzo Mirone $^{3}$ \\ ${ }^{1}$ Unità Operativa di Urologia, Ospedale Buon Consiglio - Fatebenefratelli, Napoli, Italy; \\ 2 Unità Operativa di Urologia, Casa di Cura Trusso, Ottaviano, Italy; \\ ${ }^{3}$ Clinica Urologica, Università Federico II di Napoli, Napoli; Italy.
}

\begin{abstract}
Summary Lipomas are benign mesenchymal tumours that are rarely seen in the scrotum. Few cases of primary scrotal lipomas originating from the scrotal wall have been reported in the literature. We describe the case of a giant primary intrascrotal lipoma presenting as scrotal swelling and discomfort. Findings from scrotal magnetic resonance imaging were highly suspicious for lipoma. The mass was completely excised and histological examination confirmed the diagnosis of lipoma.
\end{abstract}

KEY WORDS: Magnetic resonance imaging; Scrotal lipoma.

Submitted 22 June 2017; Accepted 19 July 2017

\section{INTRODUCTION}

Lipomas are common benign mesenchymal tumours that may occur in any part of the body. These neoplasms, however, are rarely seen in the scrotum (1). In most cases, scrotal lipomas originate from the adipose tissue of the spermatic cord evolving towards the scrotum or develop in the spermatic cord itself. Lipomas that originate from the isolated adipose lobules of the scrotal subcutaneous tissue are uncommon and are called "primary scrotal lipomas" (2-5). We describe the case of a giant primary intrascrotal lipoma presenting as swelling of the scrotum causing discomfort.

\section{Case report}

A 54-year-old man was referred to our Institution for swelling of the scrotum causing discomfort. His past medical history was unrelevant. On physical examination, an irreducible, elastic, regularly shaped mass was evident on the midline of the scrotum. Both testes and spermatic cords were found to be localized in the scrotum and were of normal consistency and size. Serum $\alpha$-feto protein, beta-human chorionic gonadotropin, and lactate dehydrogenase levels were within the normal ranges. Ultrasound evaluation showed an heterogeneous, hyperechoic solid mass, not infiltrating the testes. Magnetic resonance imaging (MRI) was required and revealed an 8 x 10 x $12 \mathrm{~cm}$ mass with homogeneous high signal intensity in Tl-weighted images. No enhancement was seen after administration of gadolinium contrast material. These findings were highly suspicious for lipoma. Surgery was planned. A longitudinal midline scrotal incision was per- formed. The testicles, epididymis and cord structures were intact. A well encapsulated, ovoid yellow-orange mass was found located within the scrotal wall and was easily removed (Figure 1). The incision was closed with no drainage and the postoperative course was uneventful. The specimen weighed $600 \mathrm{~g}$. Microscopic examination of the tissue revealed the presence of mature adipocytes and the absence of cellular atypia. Finally, the diagnosis of primary scrotal lipoma was made. Surgery provided a rapid resolution of symptoms.

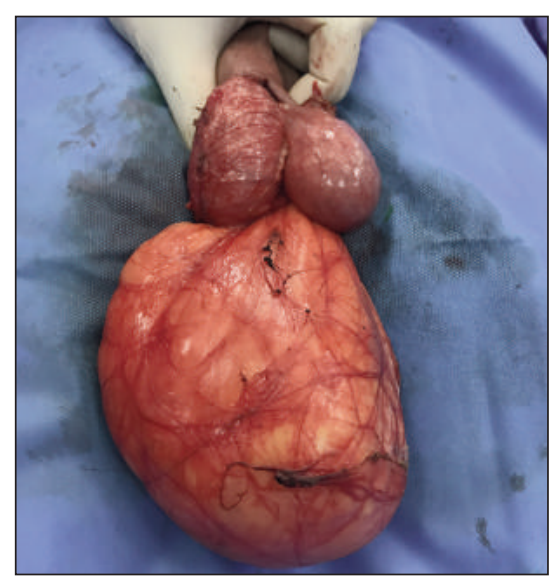

Figure 1. Intra-operative photograph showing a lobulated yellow-orange mass.

\section{Discussion}

Intrascrotal lesions provide a diagnostic challenge for the urologyst due to the multiple anatomic structures found in this confined space $(3,4)$. While most testicular lesions are malignant, the majority of tumours arising from extratesticular structures are benign (3). Despite rare, however, malignant neoplasms may also originate from extratesticular structures and include liposarcoma, leiomyosarcoma, malignant fibrous histiocytoma, rhabdomyosarcoma, mesothelioma, and lymphoma. Lipomas are the most frequent benign neoplasm of the scrotum. However, they are rarely seen in everyday clinical practice (5). These tumors may develop from the spermatic cord, from herniation of properitoneal fat or may originate from isolated fat cells of the subcutaneous tissues of the scrotal wall. In many cases, the specific site of origin cannot be easily identified. To our knowledge, there are very few cases of primary scrotal lipomas (i.e. lipomas 
originating from the scrotal wall) published in the scientific literature. Symptoms vary with mass size and pressure caused with growing. According to literature data, the size may vary considerably and weight can reach $9 \mathrm{~kg}$ (2). Ultrasonography and MRI play a pivotal role in the evaluation of scrotal masses. Ultrasonography is the the first-line imaging modality as it allows to determine whether a lesion is cystic or solid and its localization. Lipomas commonly appear as uniformly hyperechoic lesions, without internal flow at color Doppler imaging. However, ultrasound findings may be variable and are often nonspecific. MRI has the potential to narrow the diagnosic range and can be very helpful in the evaluation of lipomas as it can easily recognize fatty components. Indeed, high Tl signal intensity is characteristic of fatcontaining tumors. Moreover, lipomas can be differentiated from liposarcomas on MRI by the lack of any enhancing soft tissue. However, lesions in this location may have overlapping imaging findings (5). Sometimes, well-differentiated liposarcomas may simulate benign lipomas and the differential diagnosis is complicated (2). The present case presented with typical MRI findings thus emphasizing the diagnostic role of MRI. Surgical excision, through scrotal or combined scrotal and inguinal incision, represents the treatment of choice for symptomatic lesions or when a definitive diagnosis cannot be made at imaging (5).
Surgical excision was requird in the present case in order to manage symptoms. The surgial procedure we described was easy to peform, efficacious and safe.

\section{Conclusions}

Primary scrotal lipomas are rare, benign tumors that should be take into account in the differential diagnosis of paratesticular scrotal masses.

Surgical excision provides histopathologic diagnosis and resolution of symptoms.

\section{REFERENCES}

1. Masciovecchio S, Saldutto P, Del Rosso A, et al. An unusual case of massive funicular lipoma. Urologia. 2014; 81:184-6.

2. Kaplanoglu V, Kaplanoglu H, Parlak IS, Tatar IG. Giant intrascrotal lipoma. BMJ Case Rep. 2013; 14:2013.

3. Patel NG, Rajagopalan A, Shrotri NS. Scrotal liposarcoma - a rare extratesticular tumour. JRSM Short Rep. 2011; 2:93.

4. Montgomery JS, Bloom DA. The diagnosis and management of scrotal masses. Med Clin North Am. 2011; 95:235-44.

5. Wolfman DJ, Marko J, Gould CF, et al. Mesenchymal Extratesticular Tumors and Tumorlike Conditions: From the Radiologic Pathology Archives. Radiographics. 2015; 35:1943-54.

\section{Correspondence}

Massimiliano Creta, MD

max.creta@gmail.com

Roberto Buonopane, MD

robertobuonopane@libero.it

Sergio Di Meo, MD

s.dimeo72@gmail.com

Vittorio Imperatore, $\mathrm{MD}$

v.imperatore@alice.it

Unità Operativa di Urologia, Buon Consiglio Fatebenefratelli Hospital

Via A. Manzoni, 220, 80123, Napoli, Italy

Giacomo De Stefano, MD

drgiacomodestefano@gmail.com

Unità Operativa di Urologia, Casa di Cura Trusso

Via San Giovanni Bosco, 3, 80044 Ottaviano - NA, Italy

Ciro Barba, MD

cirobarba@yahoo.it

Ciro Imbimbo, MD

max.creta@gmail.com

Vincenzo Mirone, MD

max.creta@gmail.com

Clinica Urologica, Università Federico II di Napoli

Via S.Pansini, 5, 80131 Napoli, Italy 